\title{
Can the Desired Service Level be Achieved When the Demand and Lost Sales are Unobserved?
}

\author{
BARIŞ TAN and SELÇUK KARABATI \\ Graduate School of Business, Koç University \\ Rumeli Feneri Yolu, Sariyer, 80910 Istanbul, Turkey \\ e-mail:btan@ku.edu.tr,skarabati@ku.edu.tr
}

June 2002

In this paper we consider an inventory management problem in the retail industry with unobserved lost sales. The retailer does not know the demand for a particular product but she has access to Point-of-Sale (POS) data. The retailer uses a fixed review period, order-up-to level system to control the inventory. The objective of the retailer is to achieve a pre-specified service level. We define the service level as the fraction of demand satisfied from inventory. However, due to the unobserved lost sales nature of the problem, the retailer cannot exactly measure the current service level. We propose a POS data-based mechanism for periodic updating of the order-up-to level. We show that the periodic updating approach yields the desired service level without using any inventory information. Once the periodic updating scheme converges, it also gives the actual demand distribution.

Keywords: Retailing, Inventory Management with Lost Sales. 


\section{Can the Desired Service Level be Achieved When the Demand and Lost Sales are Unobserved?}

\section{Introduction}

Data warehouses of large companies in the retail industry store information on transactions that take place within a supply chain (sales, deliveries etc.) and the impact of these transactions on the performance indicators of the supply chain (inventory levels, time performance of deliveries etc.). On the other hand, the data warehouses do not store information on the actions of the supply chain participants that could not be transformed into "transactions" due to various problems that may arise in the supply chain. One typical example would be a customer who could not find a product she intended to buy in the shelves of the retailer. In this case, the customer's demand is unobserved, and may be lost, backordered, or substituted with another product. If the customer visits the retailer at a later point in time and buys the product, the transaction is recorded as a regular transaction without identifying its being previously backordered.

The data warehouses of retailers constitute the backbone of decision support systems such as forecasting and inventory management. Decisions that are based on the information available in the data warehouse, and made with a certain set of decision support tools, shape the next state of the information available in the data warehouse. The operational decisions such as inventory management decisions are made periodically, and this periodic nature of the decision makingtransaction recording interaction may lead to poor performance, especially when "what is not stored" in the data warehouse is not carefully taken into consideration in the decision making process.

In this paper we will focus on a certain type of data that is unobservable and therefore not recorded: unmet demand. We specifically consider the inventory management of a periodic review, order-up-to level system where the demand distribution is unknown initially and the lost sales are unobserved. As pointed out by Lariviere and Porteus (1999), in addition to the unobserved lost sales case, an inventory system may be subject to observed lost sales (as in the case of an e-retailer who does not reveal information on the availability of a certain product before it receives the order from the customer) or unobserved backordered sales (as in the case of a retailer whose customers visit the retailer until the product is available). The model presented in this paper focuses on the unobserved lost sales case.

\subsection{Problem Description and Main Result}

In this study, the question we ask is: Can the desired service level be achieved when the demand and lost sales are unobserved? We define the service level as the fraction of demand satisfied directly from inventory.

We consider inventory control of a single item subject to random stationary demand. If an item is not available in stock, the demand is not substituted with another product and lost. The 
demand distribution is not known initially. Only the Point-of-Sales (POS) data are available and on-shelf inventory data that match with the POS data are not available.

We propose a simple fixed review-period order-up-to level inventory system where the orderup-to level is updated periodically based on the current estimates obtained from the POS data collected since the last update.

Our main result is that the proposed updating mechanism that only uses the POS data converges to the desired service level in the absence of inventory and demand data. In other words, the desired service level can be achieved when the demand and lost sales are unobserved.

\subsection{The Point-of-Sales Information}

The POS information of a retailer's data warehouse displays the amount of sales of a given product that has taken place in time periods which have equal lengths, such as 15 minutes. If the sales data of a particular product shows no sales in a given time period, this may be due to unavailability of the product in the shelves of the retailer, or having no demand for that product during the time period in which the sales information is collected. If the data warehouse of the retailer tracks onhand inventory level on the shelves, then identifying the actual cause of observing no sales in a given time period can be partially identified: if on-hand inventory position is positive, no sales case is equivalent to having no demand; however, if on-hand inventory position is zero, no sales case is equivalent to either having no demand, or lost sales. This partial identification of the source of the no sales information can be a major problem in low demand items due to higher frequency of observations with no sales. Moreover, the shelf inventory position may not be reliably available in the data warehouse, in which case we cannot reach discerning conclusions as to why there is no sales in a given time period.

The POS data of a product can be analyzed at different levels of temporal granularity. In Figure 1, we present simulated weekly sales of a product over a two-year period. The average demand for the product is 96 units per week, however with a review period length of 1 day (8 hours), and order-up-to level of 13 units, the observed average weekly sales is 70.74 units. At this level of temporal granularity, the POS data do not provide too much information on the stockouts.

In Figure 2, we present POS data of the same product on an hourly basis over a period of 100 hours. At this finer level of temporal granularity, we can easily observe periods in which no transaction has taken place, however, it is still very difficult to identify lost sales instances, unless the information presented in Figure 2 is coupled with reliable shelf inventory information at the same level of detail. Storing changes in the shelf inventory for every product may be very costly, and may not be always adequate to identify every single stockout instance. Consider a product whose current shelf inventory level is one unit. A customer picks up the product and places it in her shopping cart at time 11:50. This customer checks out at time 12:10. Another customer wishes to buy the same product, checks the shelf of the product at time 11:55, and cannot find it on the shelf. The checkout of this customer is completed at time 11:58. The data warehouse of the retailer will show a positive shelf inventory level until time 12:10, and the stockout instance we have described above will never be identified. 


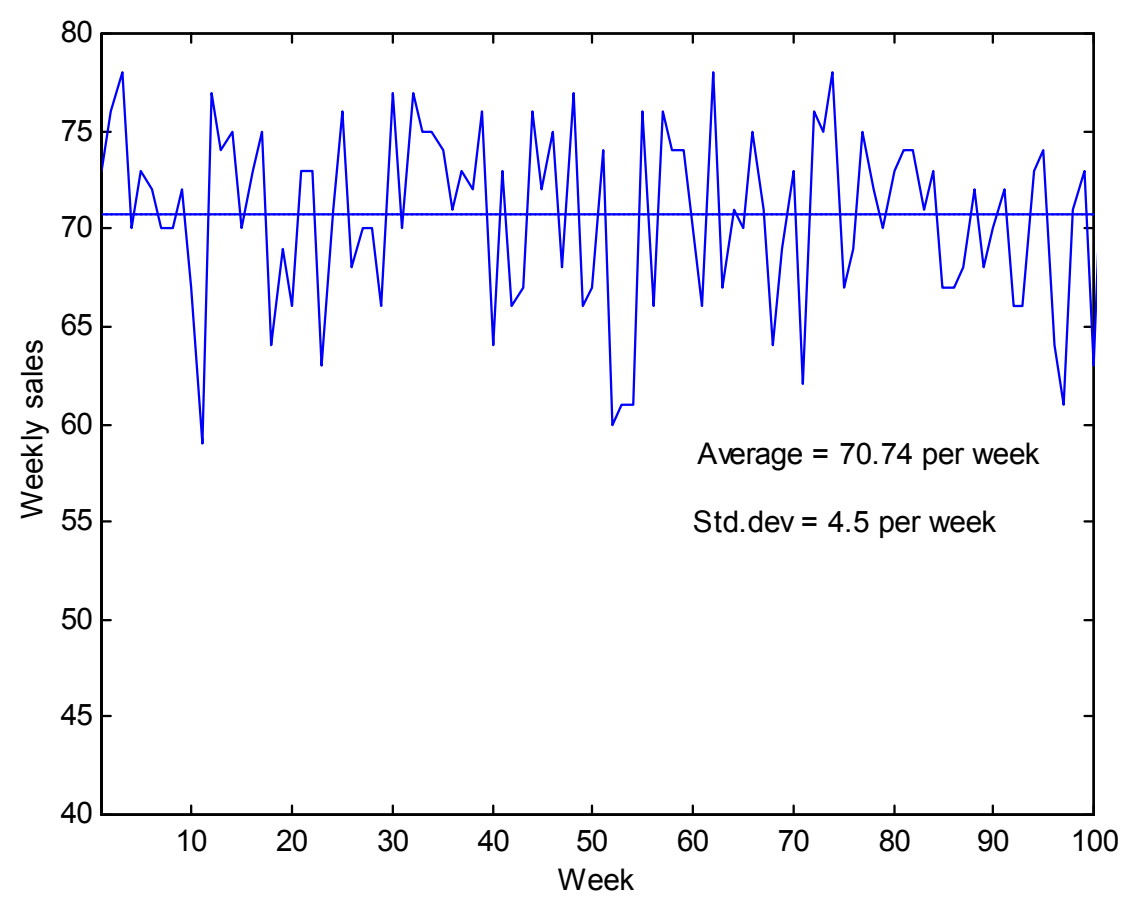

Figure 1. Simulated POS data of weekly sales. Average actual demand for the same period is 96 per week

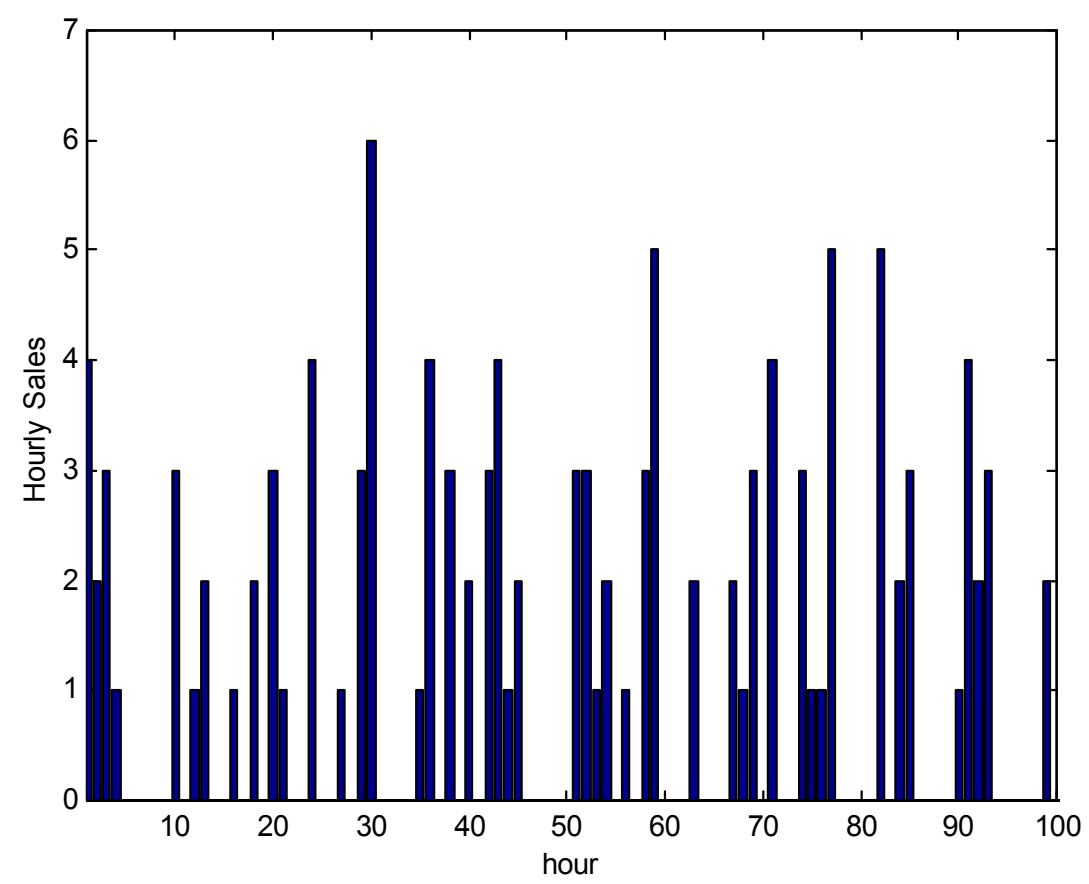

Figure 2. Simulated POS data of the hourly sales. 


\subsection{Past Work}

In the inventory management literature, there are two lines of research related to the study presented here: estimation of demand distribution in an unobservable lost sales case and dynamically updating inventory control parameters.

The literature on the estimation of demand distribution in an unobservable lost sales setting addresses a broad range of problems. Lariviere and Porteus (1999) consider a Bayesian inventory problem with unobservable lost sales. Lau and Lau (1996) develop a procedure for estimating periodic demand distribution with unobservable lost sales. Nahmias (1994) estimates the mean and variance of the demand distribution in a periodic review inventory system with lost sales. The inventory level is observed at the end of each day, and the inventory is replenished to bring its position to a predetermined order-up-to level. Therefore, only the total daily demand, which is less than the order-up-to level, is observed. Nahmias presents a comprehensive review of statistical approaches on parameter estimation for right-censored time series, and three methods to estimate the parameters of daily demand distribution from the daily sales data. Agrawal and Smith (1996) address the parameter estimation problem for the negative binomial demand distribution with lost sales. Anupindi, Dada, and Gupta (1998) suggest an approach for demand estimation in an inventory system with lost sales and substitution. This approach requires reliable inventory information that shows the stockout durations of individual products, and joint stockout durations of combination of products. Bell (2000) introduces a procedure to estimate the demand parameters for a continually solved single period inventory problem when there are stockouts.

The methods to estimate the demand distribution when the lost sales are unobserved perform well when only a small fraction of the total demand is lost. In this case, the remaining data points can be used to estimate the parameters of the demand distribution. Furthermore, these methods require frequent monitoring of the inventory to match the zero sales observations with the stockout periods. Since we consider cases where a high percentage of demand is lost and it is not possible to get corresponding inventory information continuously, these approaches do not yield satisfactory results for our case.

The issue of dynamically updating the inventory control parameters has been addressed recently in the context of dynamic forecast updating in supply chain management. These studies focus more on the adverse effects of dynamic updating. Dynamic forecast updating is considered as one of the underlying reasons of the bullwhip effect in supply chains (see for example Chen et al. (2000) and Buzacott (1999)). In our updating mechanism, the order-up-to level is updated after observing a large number of observations and the same order-up-to level is used between the updates. Therefore, the proposed updating mechanism does not introduce additional variability in the supply chain. We are not aware of any other studies that devise dynamic updating mechanisms to manage inventory systems with lost sales in the absence of demand and inventory data.

In this paper we propose an alternative approach where parameter estimation is embedded into parameter updating of a dynamic inventory management system. Suggesting an easily- 
implementable inventory control method and assuring that the desired service level can be achieved in the absence of demand and inventory information are the two main contributions of this study.

The organization of the remainder of this paper is as follows: In $\S 2$, we give the details of the model and the updating mechanism. The dynamic inventory mechanism is analyzed in $\S 3$. The convergence properties of the updating mechanism are investigated and shown in $\S 4$. Finally, we present our concluding remarks in $§ 5$.

\section{Model}

We consider inventory control of a single item subject to random and partially observed demand. If an item is not available, the demand is not substituted with another product and lost. Furthermore the lost sales case does not affect the future sales. The demand distribution is not known initially. The objective of the inventory control system is to achieve a certain pre-specified service level. In this study, the service level is defined as the fraction of demand satisfied directly from inventory. The order-up-to level used in the inventory control system is updated periodically based on the current estimates obtained from the POS data.

\subsection{Point of Sales Data and Time Scale}

In the model, there are three different time intervals. The shortest interval is the POS data collection interval. The next interval is the replenishment cycle. The POS data report the sales in equal length periods which are much smaller than the replenishment cycle. Finally, the longest time interval is the order-up-to level updating period which includes multiple replenishment cycles. The updating epochs are indexed by $t$ and the same inventory control parameters and demand estimates are used during $[t, t+1)$. There are $N$ POS data collection intervals and $N$ observations in $[t, t+1)$.

Consider the following example: the inventory level of a certain product is checked at the end of each day after the store closes, replenishment orders arrive by the next morning before the store opens, and the POS data report the total sales in 15-minute intervals. The order-up-to level is updated every 30 days based on the estimates obtained from the previous month's sales data. In this case, the different time intervals are 15 minute, one day, and 30 days. The inventory is replenished 30 times with the same order-up-to level before the order-up-to level is updated with the new estimates. Furthermore, assuming that the store is open for 12 hours/day, there are $N=(12$ hours/day)(30 days/update period)(60 minutes/hour)/(15 minute/observation $)=1440$ observations between each update and 1440 observations are used to update the estimates.

\subsection{Sales and Demand}

We assume that the demand in each POS data collection interval is an independently and identically distributed normal random variable. The mean and the standard deviation of the demand in each POS data collection interval are denoted by $\mu$ and $\sigma$. These parameters are unknown initially. 
At the updating epoch $t$, all the POS data collected since the last update are used to estimate the mean and the standard deviation of sales. The estimates of the mean and the standard deviation of the sales at time $t$ are denoted with $\hat{\mu}_{t}$ and $\hat{\sigma}_{t}$ respectively. The estimates of the mean and the standard deviation of the demand are derived form the estimates of sales and denoted with $\hat{\mu}_{t}^{\prime}$ and $\hat{\sigma}_{t}^{\prime}$ respectively.

In the retailing literature, it is noted that consumers' shopping trip may follow a weekly cycle, and shoppers' arrival rate at a store may differ by day of week and hour of day (East et al., 1994; Kahn and Schmittlein, 1989; Lam et al., 1998). In addition, retail marketing activity such as promotion also affects the demand at a given time (Walters and Rinne, 1986). However, we limit ourselves to the stationary demand case in this study. The non-stationary demand case is discussed in the conclusions and the detailed investigation of this case is left for future research.

\subsection{Inventory Control System}

The inventory system employs a fixed review period order-up-to level type control policy. Replenishment decisions are made every $R$ time units to bring the inventory position to a desired order-up-to level which is denoted by $S$. The lead time to receive an order is $L$ time units and assumed to be constant. If the lead time is greater than the review period, i.e., $L>R$, we use $L$ modulo $R$ as the replenishment lead time in the analysis, without any loss of generality. The desired fraction of demand satisfied from inventory is denoted with $\beta$.

\subsection{Periodic Updating of the Order-up-to Level}

The order-up-to level is updated periodically by using the POS data collected since the last update. The new update of the order-up-to level at the updating epoch $t$ is denoted with $S_{t}$. This order-upto level is used as the order-up-to level at each inventory replenishment until the next update.

Since the actual values of $\mu$ and $\sigma$ are unknown, the order-up-to level $S_{t}$ is computed using the estimates of $\mu$ and $\sigma, \hat{\mu}_{t}^{\prime}$ and $\hat{\sigma}_{t}^{\prime}$, and $\beta$.

The fraction of demand satisfied directly from inventory during $[t, t+1)$ is denoted with $\beta_{t}$. The updated order-up-to level yields the realized, but unobserved, service level $\beta_{t}$ during $[t, t+1)$. Consequently, at the next updating point, the estimates of the mean and the standard deviation change according to $\beta_{t}$. Although the service level itself is unobserved, its impact on the sales process will be observed in the POS data. Our POS data-based updating scheme uses the changes observed in the POS data to update the estimates of the mean and the standard deviation of the demand process. This procedure is repeated until the estimates do not change from one updating point to the next.

Figure 3 shows the sequence and the relationship between the updating period, the review period and $t$. 


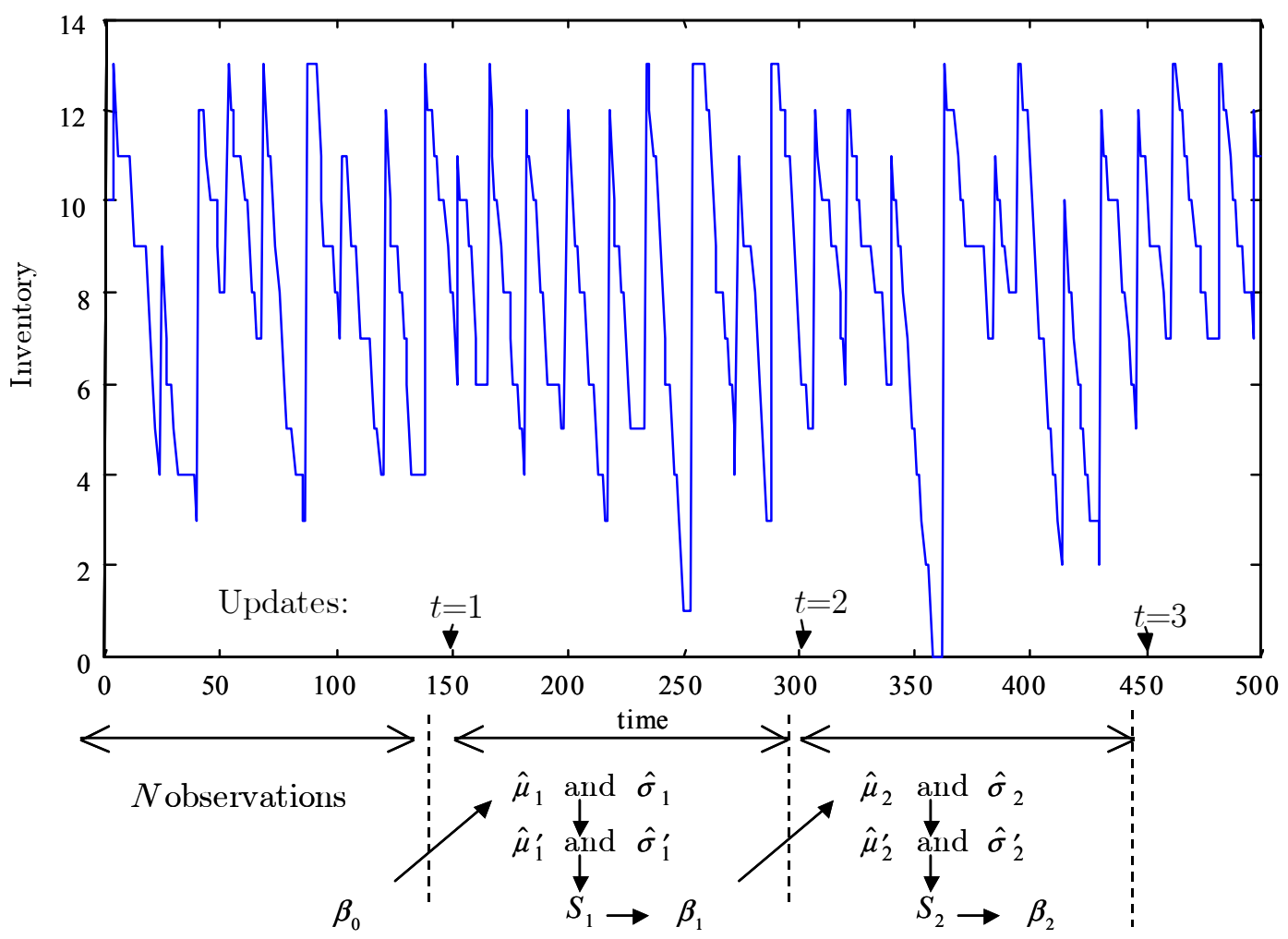

Figure 3. The sequence of actions, review, and update periods

\section{Operation and Analysis of the Updating Mechanism}

In order to analyze the updating mechanism analytically, we study the function that relates the previous service level $\beta_{t-1}$ to the one that is realized after updating the order-up-to level that is $\beta_{t}$. Let $g\left(\beta_{t-1}\right)$ be the function that relates the previous service level $\beta_{t-1}$ to the next service level $\beta_{t}$.

In order to determine the function $g$, we first analyze the effect of the previous update on the estimates. Namely, we first study the effect of the resulting service level $\beta_{t-1}$ on the estimates of sales mean and standard deviation at the updating epoch $t, \hat{\mu}_{t}, \hat{\sigma}_{t}$. Next, we discuss how the estimates for the mean and standard deviation of demand, $\hat{\mu}_{t}^{\prime}$ and $\hat{\sigma}_{t}^{\prime}$, are generated from the sales estimates. The order-up-to level $S_{t}$ can be updated by using the new demand estimates. Finally, we investigate the effect of the updated order-up-to level on the next service level that will be realized $\beta_{t}$. Figure 4 shows the order of analysis which is followed in this section.

$$
\left.\boldsymbol{\beta}_{t-1} \quad \mathrm{a}\right) \rightarrow \hat{\mu}_{t}, \hat{\sigma}_{t}-(\mathrm{b}) \rightarrow \quad \hat{\mu}_{t}^{\prime}, \hat{\sigma}_{t}^{\prime}-(\mathrm{c}) \rightarrow S_{t}-(\mathrm{d}) \rightarrow \boldsymbol{\beta}_{\boldsymbol{t}}
$$

Figure 4. Analysis of the Updating Mechanism 


\subsection{Estimation of the Sales Mean and Standard Deviation (a)}

Let us consider the updating epoch $t$ where the new mean and standard deviation of the sales are to be estimated. Once the POS data during $[t-1, t)$ are available, the new mean and standard deviation of sales, $\hat{\mu}_{t}$ and $\hat{\sigma}_{t}$ are estimated directly by using $N$ observations. Let the $N$ observations during $[t-1, t)$ be re-indexed from 1 to $N$. Let $s_{i}$ be the sales in POS data collection period $i$. Then the estimate of the mean and standard deviation of sales in each POS data collection period are obtained as

$$
\begin{aligned}
& \hat{\mu}_{t}=\frac{1}{N} \sum_{i=1}^{N} s_{i} . \\
& \hat{\sigma}_{t}=\sqrt{\frac{1}{N-1} \sum_{i=1}^{N}\left(s_{i}-\hat{\mu}_{t}\right)^{2}},
\end{aligned}
$$

If there are some stockout periods, these periods are recorded as zero sales in the data warehouse, i.e., $s_{i}=0$ for some $i$. As a result, the estimated sales mean is lower than the demand mean, $\hat{\mu}_{t}<\mu$, and the estimated standard deviation of the sales is greater than the standard deviation of the demand $\hat{\sigma}_{t}>\sigma$.

The deviation between the actual demand parameters and the estimated sales parameters depend on the previous realized service level $\beta_{t-1}$. The previous service level is not known because the actual demand is never fully observed. The following proposition shows how $\hat{\mu}_{t}$ and $\hat{\sigma}_{t}$ are related to $\mu, \sigma$, and $\beta_{t-1}$.

Proposition 1:

$$
\begin{aligned}
& \hat{\mu}_{t} \cong \mu \beta_{t-1}, \\
& \hat{\sigma}_{t}^{2} \cong \sigma^{2} \beta_{t-1}+\mu^{2} \beta_{t-1}\left(1-\beta_{t-1}\right),
\end{aligned}
$$

The proof of Proposition 1 is provided in the Appendix.

\subsection{Estimation of the Demand Mean and Standard Deviation (b)}

Since $\hat{\mu}_{t}$ underestimates $\mu$ and $\hat{\sigma}_{t}$ overestimates $\sigma$ due to possible stockout periods that are recorded as zero sales, the sales estimates need to be adjusted to generate the estimates for the demand.

Had we known the actual service level $\beta_{t-1}$, the actual mean and the standard deviation of the demand could have been directly estimated from Equations (3) and (4), by using $\beta_{t-1}$, $\hat{\mu}_{t}$ and $\hat{\sigma}_{t}$. Since the actual service level is unknown, we generate the parameter estimates of the demand process by approximating the actual service level $\beta_{t-1}$ with the desired service level $\beta$. The estimates 
for the mean and standard deviation of the demand, $\hat{\mu}_{t}^{\prime}$ and $\hat{\sigma}_{t}^{\prime}$, are now computed from Equations (1) and (2) as

$$
\begin{aligned}
& \hat{\mu}_{t}^{\prime}=\frac{\hat{\mu}_{t}}{\beta} \\
& \hat{\sigma}_{t}^{\prime}=\sqrt{\frac{\hat{\sigma}_{t}^{2}}{\beta}-\frac{\hat{\mu}_{t}^{2}(1-\beta)}{\beta^{2}}}
\end{aligned}
$$

In the above equations $\hat{\mu}_{t}$ and $\hat{\sigma}_{t}$ are obtained from the POS data, and $\beta$ is given as a management constant. Since this method is employed when there are unobserved lost sales and therefore the actual service level is lower than the desired service level, the term inside the square root in Equation (6) is non-negative. If the term inside the square root is negative, it indicates that the current service level is above the desired level and there is no need to adjust the estimates. In this case, we simply use $\hat{\mu}_{t}^{\prime}=\hat{\mu}_{t}$ and $\hat{\sigma}_{t}^{\prime}=\hat{\sigma}_{t}$.

In order to analyze the updating mechanism, the above equations can also be written in terms of $\mu, \sigma$, and $\beta_{t-1}$. Inserting Equations (3) and (4) into Equations (5) and (6) yields

$$
\begin{aligned}
& \hat{\mu}_{t}^{\prime}=\frac{\hat{\mu}_{t}}{\beta}=\mu \frac{\beta_{t-1}}{\beta}, \\
& \hat{\sigma}_{t}^{\prime}=\sqrt{\frac{\hat{\sigma}_{t}^{2}}{\beta}-\frac{\hat{\mu}_{t}^{2}(1-\beta)}{\beta^{2}}}=\sigma \frac{\sqrt{\beta_{t-1}}}{\beta c v} \sqrt{\beta\left(c v^{2}+1\right)-\beta_{t-1}},
\end{aligned}
$$

where $c v=\sigma / \mu$ is the coefficient of variation of demand per period.

\subsection{Updating the Order-up-to Level (c)}

In a periodic review, order-up-to level inventory system with lost sales and normal demand, the order-up-to level is determined for a given desired service level $\beta$ as (see Silver et al., Chapter 7):

$$
S=\mu(R+L)+z_{\beta} \sigma \sqrt{R+L}
$$

In this equation the safety stock multiplier $z_{\beta}$ satisfies

$$
\eta\left(z_{\beta}\right)=\frac{(1-\beta) \mu R}{\beta \sigma \sqrt{R+L}},
$$

where $\eta(z)$ is the expected number of units short in each cycle. When the distribution of demand during lead time and the review time is standard normal, $\eta(z)$ can be determined as

$$
\eta(z)=\int_{z}^{\infty}(x-z) \frac{1}{\sqrt{2 \pi}} e^{-\frac{1}{2} x^{2}} d x=\phi(z)-z \Phi(z) .
$$


where $\phi(z)$ and $\Phi(z)$ are the density function and cumulative distribution function of the standard normal given as

$$
\phi(z)=\frac{1}{\sqrt{2 \pi}} e^{-\frac{1}{2} z^{2}} \text { and } \Phi(z)=\int_{z}^{\infty} \phi(x) d x .
$$

Note that, in the derivation of Equation (10), the lost sales from a previous cycle that are not met at the start of the next cycle are double-counted (Silver et al., 1988). As a result Equation (10) is an approximation and it is quite accurate when $\beta$ is close to 1.

Since the actual mean and the standard deviation of the demand are not known at the time the order-up-to level is updated, the estimates of the mean and the standard deviation of demand are used. Let $z_{t}$ be the safety stock multiplier that is set at the updating epoch $t$ by using the POSbased estimates of the demand. Then $z_{t}$ is determined from

$$
\eta\left(z_{t}\right)=\frac{(1-\beta) \hat{\mu}_{t}^{\prime} R}{\beta \hat{\sigma}_{t}^{\prime} \sqrt{R+L}}
$$

and the order-up-to level is updated as

$$
S_{t}=\hat{\mu}_{t}^{\prime}(R+L)+z_{t} \hat{\sigma}_{t}^{\prime} \sqrt{R+L} .
$$

By using Equation (7) and (8), the above equation can also be written in terms of $\mu, \sigma$, and $\beta_{t-1}$ as

$$
S_{t}=\mu \frac{\beta_{t-1}}{\beta}(R+L)+z_{t} \sigma \frac{\sqrt{R+L}}{\beta c v} \sqrt{\beta_{t-1} \beta\left(c v^{2}+1\right)-\beta_{t-1}^{2}}
$$

\subsection{Resulting Service Level $\boldsymbol{\beta}_{t}$ (d)}

The order-up to level set during a given iteration of the updating mechanism, $S_{t}$, determines the next service level. As long as the estimates of the mean and the standard deviation differ from the actual mean and the standard deviation of the demand, the resulting service level $\beta_{t}$ in the period $[t, t+1)$ will not be equal to the desired service level $\beta$. In practice the desired service levels are very high and usually greater than $90 \%$. In this range, since approximation (10) is very accurate, we can claim that if the estimates of mean and standard deviation do match the actual mean and standard deviation, then the resulting service level calculated from Equation (12) will be equal to the desired service level. Note that our accuracy assumption is the standard assumption in the inventory management literature.

On the other hand when the estimates of the mean and the standard deviation differ from the actual values, in order to analyze the updating mechanism it is necessary to determine the service level exactly.

Figure 5 shows a sample realization of the inventory level for a specific system. Let $I_{1}$ be the inventory level when the inventory is reviewed. According to the order-up-to level inventory control policy, an order of size $Q=S_{t}-I_{1}$ is placed at this time. Let $I_{2}$ be the inventory level just after 
the order is received and $I_{3}$ be the inventory level just before the order is received. Due to random demand, $I_{1}, I_{2}$, and $I_{3}$ are random variables.

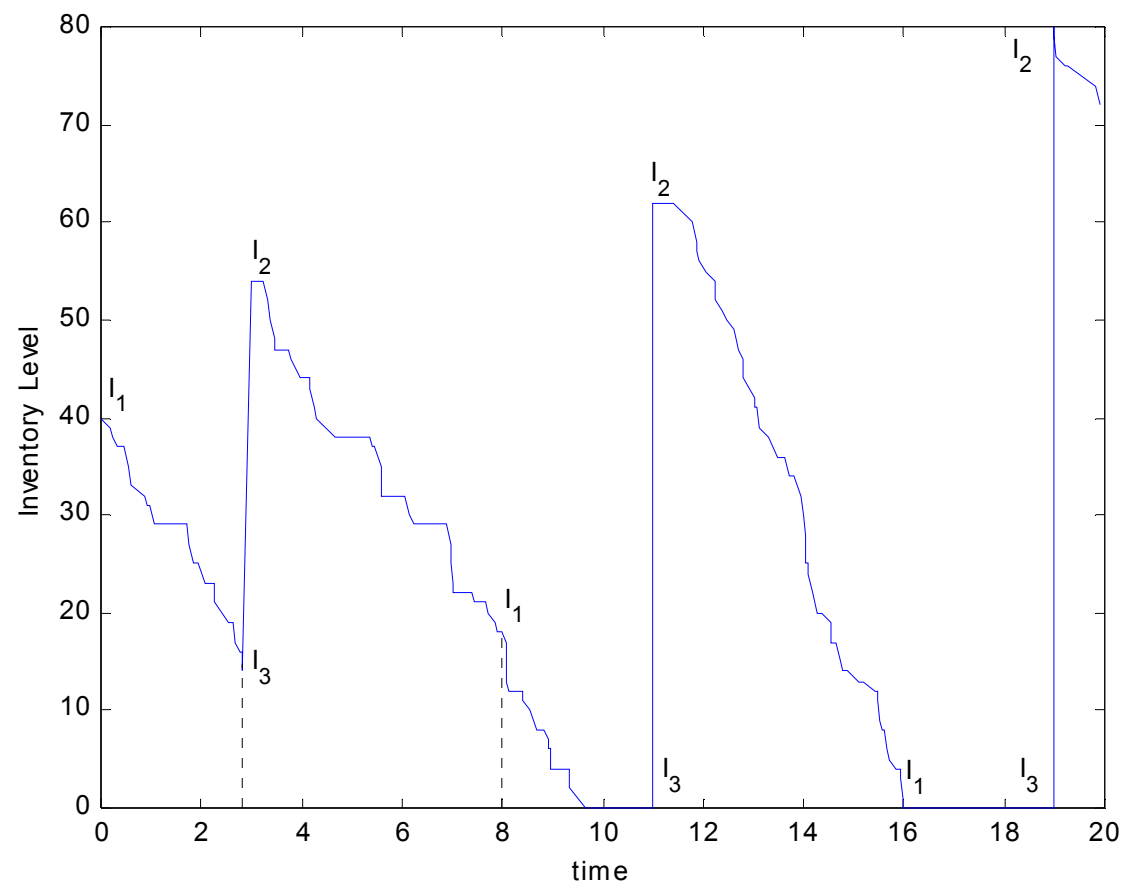

Figure 5. An inventory system with $S_{t}=80, R=8$, and $L=3$.

Let $X, Y, Z$ be random variables that represent the demand over the review period, over the lead time, and over the period from delivery of an order until the next review respectively. Since the demand in each period is an i.i.d. normal random variable with mean $\mu$ and standard deviation $\sigma, X, Y$, and $Z$ are also normal random variables with means $\mu R, \mu L, \mu(R-L)$, and standard deviations $\sigma \sqrt{R}, \sigma \sqrt{L}, \sigma \sqrt{R-L}$ respectively.

Now consider a period of length $R$ from the instance an order is received until the delivery of the next order (for example, from time 3 to time 11 in Figure 5). Let $N_{S}$ be the number of units short during this review period. Since the starting inventory of this period is $I_{2}$, the number of units short is

$$
N_{S}=\left(X-I_{2}\right)^{+}
$$

where $(x)^{+}=\max \{0, x\}$. Therefore the fraction of demand satisfied directly from shelf is (Silver et al., 1988):

$$
\boldsymbol{\beta}_{t}=1-\frac{E\left[N_{S}\right]}{E[Q]+E\left[N_{S}\right]}
$$

By considering Figure 5 the following equalities can be written:

$$
Q=S_{t}-I_{1},
$$




$$
\begin{aligned}
& I_{1}=\left(I_{2}-Z\right)^{+}, \\
& I_{2}=\left(S_{t}-Y\right)^{+}, \\
& I_{3}=\left(I_{1}-Y\right)^{+} .
\end{aligned}
$$

Now, inserting Equation (19) into Equation (15) yields the following expression for $N_{S}$

$$
N_{S}=\left(X-\left(S_{t}-Y\right)^{+}\right)^{+}=\left\{\begin{array}{cc}
X+Y-S_{t} & X+Y \geq S_{t}, S_{t} \geq Y \\
X & X \geq 0, S_{t}<Y \\
0 & \text { otherwise }
\end{array},\right.
$$

The expectation of $N_{S}$ can be evaluated as

$$
\begin{aligned}
E\left[N_{S}\right] & =\int_{0}^{S_{t}} \int_{S_{t}-y}^{\infty}\left(x+y-S_{t}\right) f_{X}(x) f_{Y}(y) d x d y+\int_{S_{t}}^{\infty} \int_{0}^{\infty} x f_{X}(x) f_{Y}(y) d x d y \\
& =\int_{0}^{S_{t}} \int_{S_{t}-y}^{\infty}\left(x+y-S_{t}\right) f_{X}(x) f_{Y}(y) d x d y+\mu R \Phi\left(\frac{S_{t}-\mu L}{\sigma \sqrt{L}}\right)
\end{aligned}
$$

where $f_{X}(x), f_{Y}(y), f_{Z}(z)$ are the probability density functions of $X, Y$, and $Z$.

Similarly, by inserting Equation (19) into (18), $I_{1}$ can be written as

$$
I_{1}=\left(\left(S_{t}-Y\right)^{+}-Z\right)^{+}=\left\{\begin{array}{cc}
S_{t}-Y-Z & S_{t} \geq Y, S_{t} \geq Y+Z \\
0 & \text { otherwise }
\end{array}\right.
$$

The expectation of $I_{1}$ is evaluated as

$$
E\left[I_{1}\right]=\int_{0}^{S_{t}} \int_{0}^{S_{t}-y}\left(S_{t}-y-z\right) f_{Z}(z) f_{Y}(y) d z d y
$$

Finally, the expected order size can be determined from Equation (17) as

$$
E[Q]=S_{t^{-}} E\left[I_{1}\right]
$$

Since $E[Q]$ and $E\left[N_{S}\right]$ are determined, Equation (16) yields $\beta_{t}$. Using the expression of $S_{t}$ in terms of $\mu, \sigma$, and $\beta_{t-1}$ given in Equation (14) in integrals (22) and (24) with Equation (16) gives the function $g\left(\beta_{t-1}\right)$.

For normally distributed random variables, the integrals (22) and (24) do not yield closed form expressions. But they can be evaluated numerically. In order to study the properties of the updating mechanism, a lower bound approximation for $g\left(\beta_{t-1}\right)$ that allows an analytical investigation can be used. In the next section, we derive a lower bound approximation for $g\left(\beta_{t-1}\right)$. 


\subsection{A Lower Bound for the Resulting Service Level}

Let $h\left(\beta_{t-1}\right)$ be the lower bound approximation of $g\left(\beta_{t-1}\right)$. In order to derive $h\left(\beta_{t-1}\right)$, let us first replace the random variables by their expected values in Equations (17), (18), (19), and (20) by employing the mean-value analysis. Note that, since $E[\max \{X, 0\}] \geq \max \{E[X], 0\}$ following the Jensen's inequality, this approach yields a lower bound for the exact expected value. These four equations define a set of equations for four unknown approximate expected values.

Let $E[\underline{Q}]$ be the approximation of $E[Q]$. Reducing Equations (17), (18), (19), and (20) for $E[\underline{Q}]$ yields

$$
\left(\left(S_{t}-E[\underline{Q}]-\mu L\right)^{+}+E[\underline{Q}]-\mu(R-L)\right)^{+}=S_{t}-E[\underline{Q}]
$$

The above equation is solved for $E[Q]$ as

$$
E[\underline{Q}]=\left\{\begin{array}{cc}
\mu R & \mu(R+L)<S_{t} \\
\frac{1}{2}\left(S_{t}+\mu(R-L)\right) & \mu(R-L) \leq S_{t}<\mu(R+L) \\
S_{t} & S_{t}<\mu(R-L)
\end{array}\right.
$$

The above result is intuitive: for large values of $S_{t}$, the expected order size is $\mu R$ which is the expected demand over the review period. For small values of $S_{t}$, the demand over the lead time is more likely to exceed $S_{t}$ and the demand over the review period is more likely to exceed the order size. This means that the on hand inventory is expected to be zero when the next order is placed. Consequently, the expected order size is equal to the order-up-to level $S_{t}$. For intermediate values of $S_{t}$, the above equation suggests a linear interpolation between these two regions.

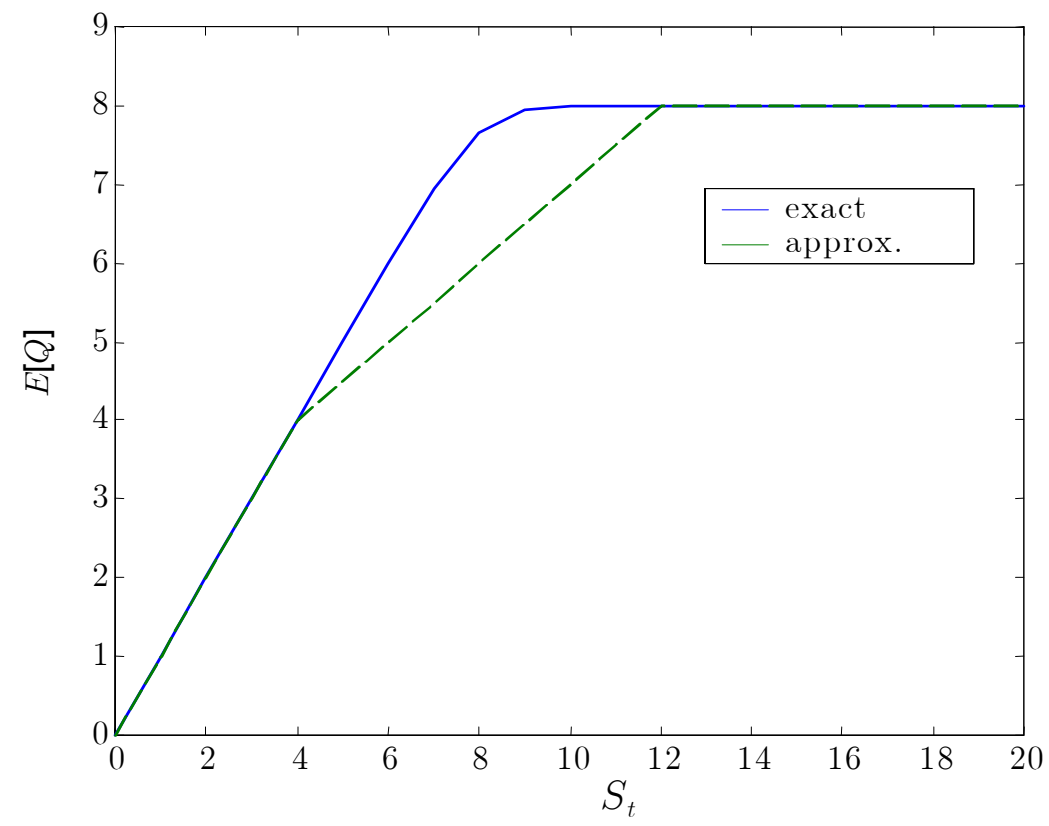

Figure 6. Exact and Approximate Order Size vs. $S_{t}$ 
Figure 6 compares the exact expected order size that is calculated numerically from Equations (24) and (25) with the approximation given in Equation (27) for a specific case. As the figure shows, $E[\underline{Q}]$ is approximately equal to $E[Q]$ for large and small values of $S$ and a lower bound for intermediate values.

Similarly, let $E\left[\underline{N}_{s}\right]$ be the approximation of $E\left[N_{S}\right]$ that is obtained by considering the expected number of units short during the review and the lead time. This approximation is the one that is employed in the derivation of Equation (10) and gives an upper bound due to double counting the shortages from the point an order is placed until it is received. Namely,

$$
E\left[\underline{N}_{s}\right]=\sigma \sqrt{R+L} \eta\left(\underline{z}_{t}\right)
$$

where $\underline{z}_{t}=\frac{S_{t}-\mu(R+L)}{\sigma \sqrt{R+L}}$.

Finally, an approximate value for the resulting service level, $\beta_{t}$, can be determined by using $E[\underline{Q}]$ and $E\left[\underline{N}_{s}\right]$ in Equation (16):

$$
\underline{\beta}_{t}=h\left(\underline{\beta}_{t-1}\right)=\frac{E[\underline{Q}]}{E[\underline{Q}]+E\left[\underline{N}_{s}\right]}
$$

Since $E[\underline{Q}] \leq E[Q]$ and $E\left[\underline{N}_{s}\right] \geq E\left[N_{S}\right]$, the above approximation is a lower bound for the exact service level $\beta_{t}$, i.e., $\underline{\beta}_{t} \leq \beta_{t}$.

The lower bound approximation of $g$ can be written explicitly by inserting Equations (27) and (28) into Equation (29):

$$
\underline{\beta}_{t}=h\left(\beta_{t-1}\right)=\left\{\begin{array}{cc}
\frac{\mu R}{\mu R+\sigma \sqrt{R+L} \eta\left(\underline{z}_{t}\right)} & \mu(R+L)<S_{t} \\
\frac{S_{t}+\mu(R-L)}{S_{t}+\mu(R-L)+2 \sigma \sqrt{R+L} \eta\left(\underline{z}_{t}\right)} & \mu(R-L) \leq S<\mu(R+L) \\
\frac{S_{t}}{S_{t}+\sigma \sqrt{R+L} \eta\left(\underline{z}_{t}\right)} & S_{t}<\mu(R-L)
\end{array}\right.
$$

\section{Convergence of the Updating Mechanism}

The function $g\left(\beta_{t-1}\right)$ that relates the previous service level $\beta_{t-1}$ to the next service level $\beta_{t}$ is defined through the order-up-to level relationship given in Equation (14) and the resulting service level given in Equation (16). Given that the updating mechanism converges, the convergence point(s) can be determined by solving equation $x=g(x)$. Due to the complexity of this function, the convergence points can only be determined numerically. However, our objective is not to determine the convergence points, but to show that the updating mechanism converges to the desired fraction 
of demand satisfied directly from demand. We first investigate the convergence properties of $g\left(\beta_{t-1}\right)$ numerically.

\subsection{A Numerical Investigation}

Figure 7 shows the relationship between $\beta_{t-1}$ and $\beta_{t}$ for a specific case. This relationship is established by numerically evaluating integrals given in Equation (22) and (24) with Equations (14) and (16). In Figure 7; the dashed line is the $\boldsymbol{\beta}_{t}=\boldsymbol{\beta}_{t-1}$ line and the solid curve is the function $g\left(\boldsymbol{\beta}_{t-1}\right)$. The intersection points of these two lines are the solutions to $\beta_{t}=g\left(\boldsymbol{\beta}_{t-1}\right)$.

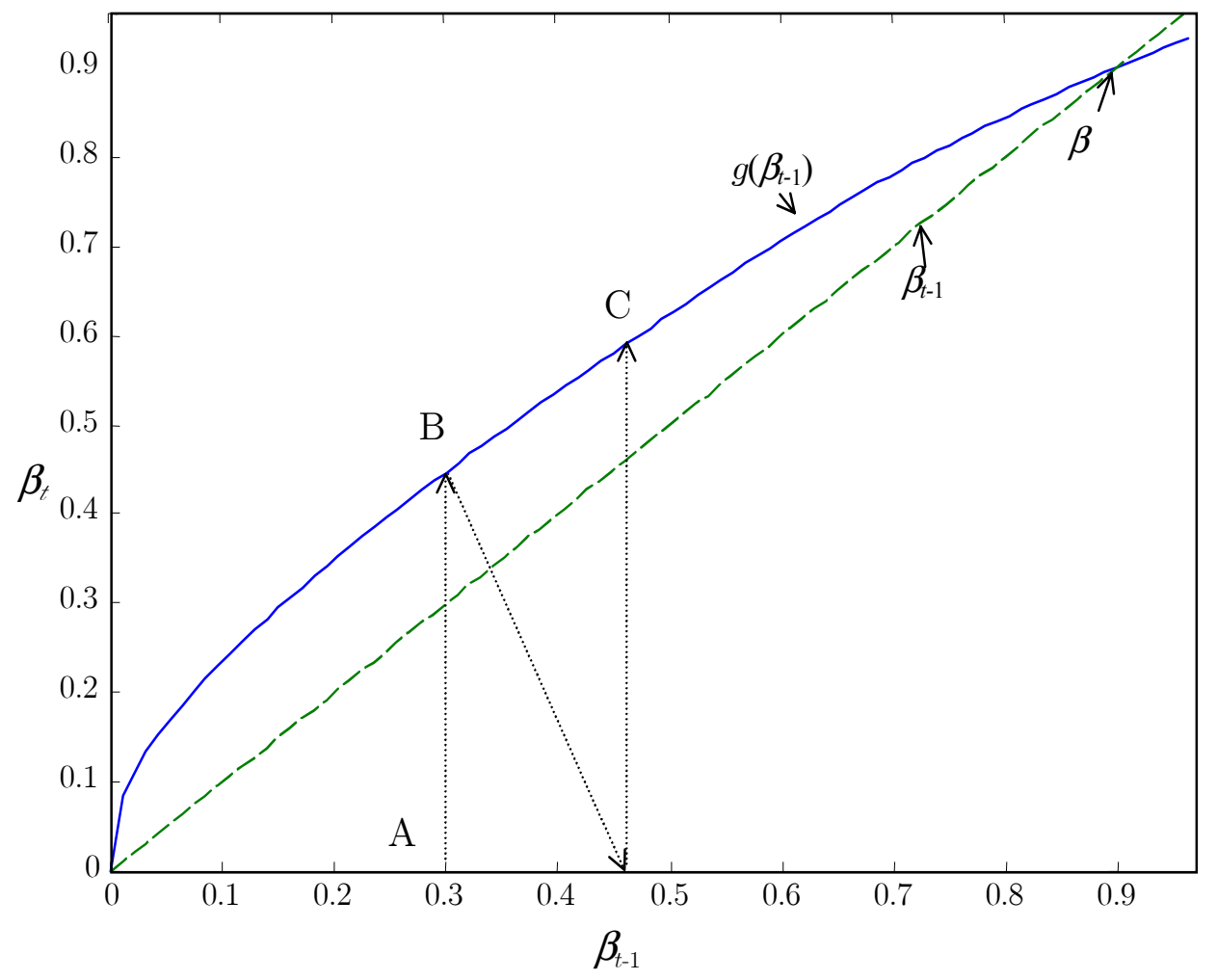

Figure 7. Convergence of the updating mechanism: $R=6, L=1, \mu=2 \sigma^{2}=0.4, \beta=0.9$.

As the figure shows two curves intersect at two points: at the origin and also at $\beta_{t-1}=0.90$ which is the desired service level. These are the only solutions to $\beta_{t}=g\left(\boldsymbol{\beta}_{t-1}\right)$. When $g\left(\beta_{t-1}\right)$ is above the $\beta_{t}=\beta_{t-1}$ line, the next service level will be higher after the update, i.e., $\boldsymbol{\beta}_{t}>\boldsymbol{\beta}_{t-1}$. However, when it is below, the next service level will be lower than the current service level, i.e., $\beta_{t}<\beta_{t-1}$. Therefore, if the updating mechanism starts at an initial level that is higher than 0.90 , the service level gradually decreases and converges to 0.90. Similarly, if it starts at a level between 0 and 0.90, it gradually increases and again converges to 0.90 . Then $\beta_{t-1}=0.90$ is the only convergence point. Figure 7 shows that starting with a service level of $30 \%$ (point A), the updating mechanism improves the service level to $46 \%$ (point B) in the next iteration and to $60.5 \%$ (point $\mathrm{C}$ ) in the following iteration. 

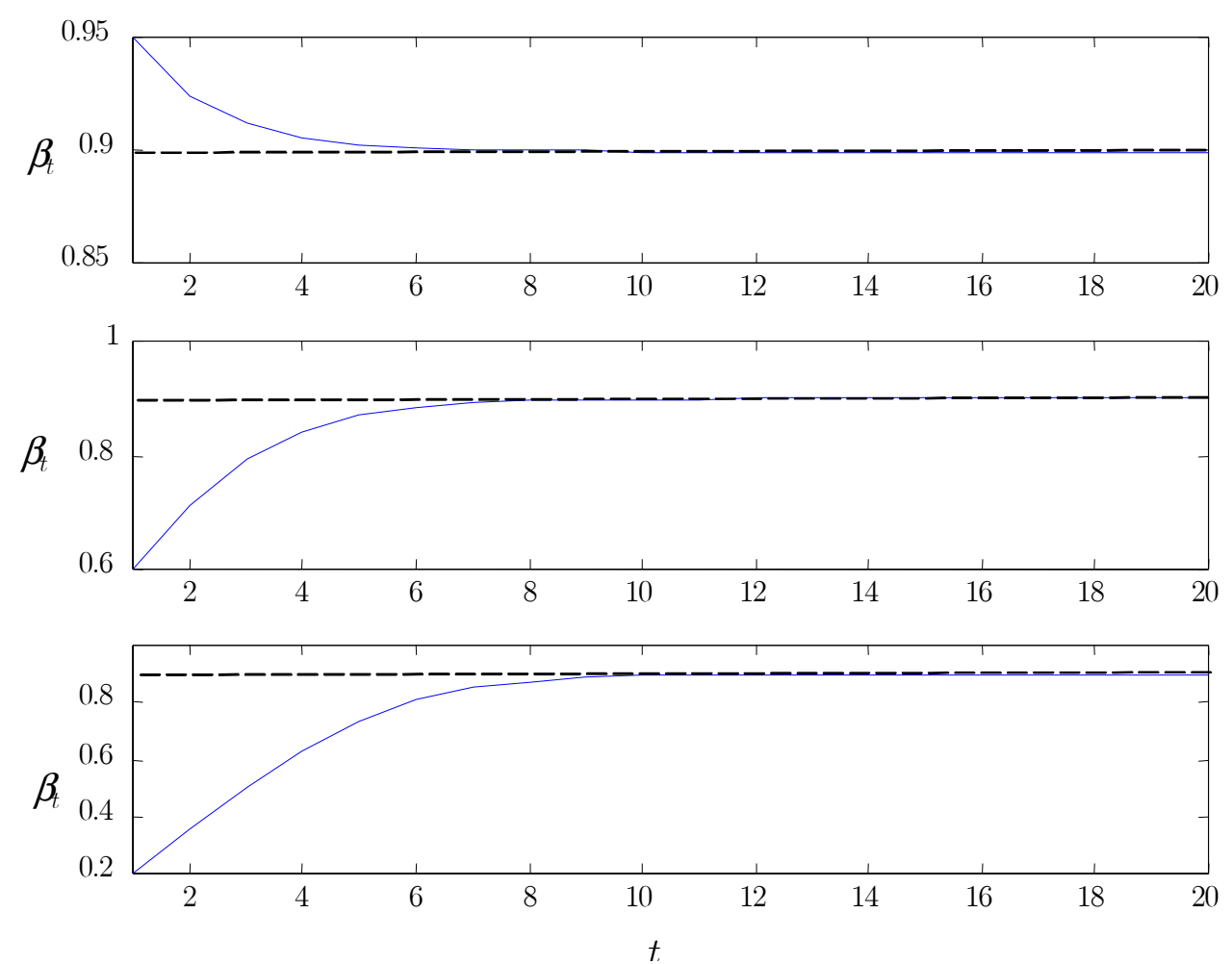

Figure 8. Convergence of the service level: $R=6, L=1, \mu=2 \sigma^{2}=0.4$, and $\beta=0.9$
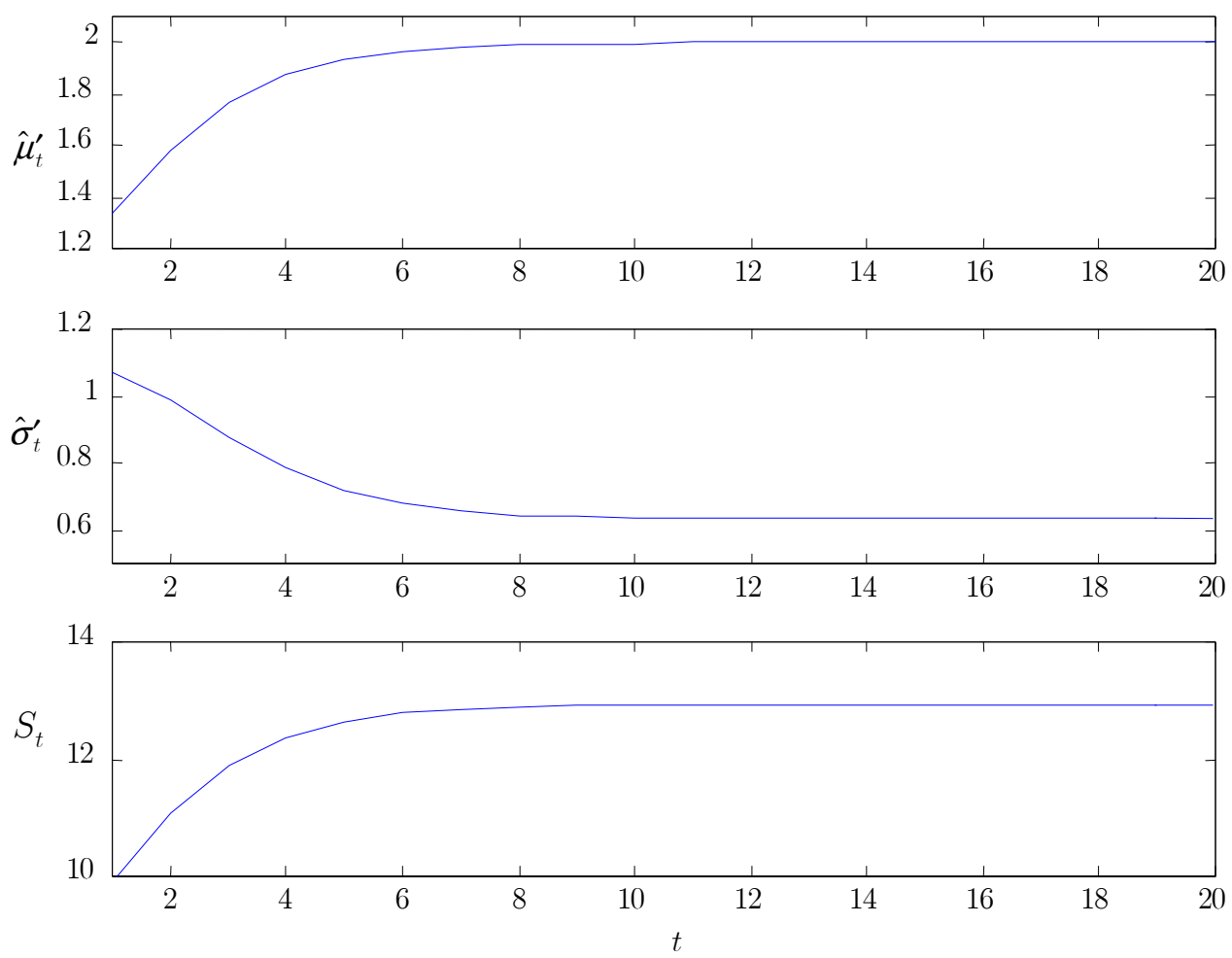

Figure 9. Convergence of the mean, standard deviation, and order-up-to level: $R=6, L=1, \mu=2$ $\sigma^{2}=0.4, \beta=0.9, \beta_{0}=0.6$ 
Figure 8 illustrates how the updating mechanism iterates starting with a service level of $95 \%$, $60 \%$, and $20 \%$ respectively for this specific case. As these plots show, this mechanism yields the desired service level of $90 \%$ after a couple of iterations for all three starting levels. For the same example, Figure 9 shows the convergence of the mean and standard deviation estimates and the calculated order-up-to level as the updating mechanism iterates for a starting service level of $60 \%$. The estimates of the mean and the standard deviation of demand converge to the actual mean and the standard deviation of demand as the updating mechanism iterates.

\subsection{Properties of the Updating Function}

After considering these numerical examples, we now present some properties of the updating mechanism to derive insights as to when convergence is guaranteed. All the proofs of these properties are given in the Appendix. The first proposition shows that the origin and the desired service level are possible convergence points.

\section{Proposition 2:}
a. $g(\beta)=\beta$
b. $g(0)=0$.

Proposition 2 shows that the desired service level is one of the convergence points. Depending on the initial service level, the updating mechanism can yield the desired service level. Next, we investigate how the function $g\left(\beta_{t-1}\right)$ reaches $g(\beta)=\beta$ starting at $g(0)=0$. The next proposition states that $g(x)$ is an increasing function in an interval starting at the origin.

\section{Proposition 3:}

$g(x)$ is either an increasing function of $x$ for all $x$, or it is an increasing function for $\beta>x>0$ and decreasing function $x>\beta^{\prime}$ where $\beta^{\prime}$ can be determined numerically for a given system.

The proof establishes that $g(x)$ is an increasing function between 0 and a specific point which can be determined numerically for a given system. Moreover, as it will be seen, the numerical determination of this specific point is not required to study the convergence properties of the updating mechanism.

In order to show the convergence of the updating mechanism to the desired service level, we also need to study the concavity of $g(x)$. However, investigating the concavity of $g(x)$ directly is not tractable due to the complexity of $g(x)$. Therefore, we examine the properties of its lower bound $h(x)$. The next proposition is related to the properties of $h(x)$ :

Proposition 4: Properties of the lower bound $h(x)$

a. $h(\beta)=\beta$

b. $h(0)=0$

c. $h(x)$ is either an increasing function of $x$ for all $x$, or it is an increasing function for $\beta>x>0$ and decreasing function $x>\beta$. 
d. $h(x)$ is a concave function of $x$ for $\beta^{\prime \prime}>x>0$ and convex function of $x$ for $x>\beta^{\prime \prime}$ where $\beta^{\prime \prime}$ can be determined numerically for a specific system

Proposition 4 shows that the lower bound of $g(x)$ exhibits the same properties as $g(x)$. In addition to these properties, $h(x)$ is also a concave function between 0 and a specific point which can be determined numerically for a given system. The numerical value of this specific point is not required.

Finally, the above propositions allow us to state our main result that is the updating mechanism always converges to the desired level. In other words, the desired service level can be achieved when the demand lost sales are unobserved.

\section{Proposition 5:}

The updating mechanism converges to a unique point which is the desired service

level, starting at any given initial level.

The proof of the main result is also given in the Appendix.

\subsection{Simulation}

The updating mechanism presented in the previous sections is analyzed under a number of assumptions. Especially, assuming normally distributed demand and requiring that the length of each POS data collection period is to be very small compared to the length of the update period may affect the performance of the mechanism in an actual application.

In order to check the validity of the proposed approach, we simulate an inventory system with non-normal demand and longer POS data collection period. We use the updating mechanism to investigate the effects of relaxing the assumptions on the performance.

In the simulation, customers arrive according to a Poisson process with a mean of 2 per hour. When a customer arrives, she purchases a single item with $1 / 3$ probability and purchases two items with probability $1 / 3$. She leaves the store without any purchase with probability $1 / 3$. This information is not known to the inventory manager and therefore not used in the periodic updating mechanism.

The transaction data are combined in hourly POS reports. That is, only the total hourly sales information is available. The inventory is checked at the end of each day, i.e., once every 8 hours, and additional items are ordered to bring the inventory position to the current desired order-up-to level. The lead time to receive the orders is 1 hour. The order-up-to level is updated once a month by using the previous month's POS data based on Equations (12) and (13). We assume that the desired service level is $90 \%$.

Figure 10 depicts the realized service level at each iteration for this system. The solid line represents the mean service level and the dotted lines are the upper and lower $95 \%$ lines for 100 replications of the simulation. As the figure shows, regardless of the relaxed assumptions, the updating mechanism yields a service level which is slightly higher than the desired level after a couple of updates. 
There are two main reasons for the deviation of the simulation results and the theoretical results presented in the preceding sections. First, since the POS data give the hourly sales, at each observation point, the number of items sold may be greater than one. In this case, it is possible to partially satisfy the demand. As a result, the argument that relates the service level to the time average is not an exact relationship and this introduces an error into Equations (3) and (4). Furthermore, calculation of the multiplier in the order-up-to level uses the assumption that the demand is normally distributed. In this example, the compound distribution of hourly sales cannot be approximated well by a normal distribution. Regardless of these differences in the simulation model, the obtained results show that the proposed approach is robust to variations in its most critical assumptions and yields the desired service level after only two updates starting with a service level of $78 \%$. When the updating mechanism converges, the mean and the standard deviation of demand are also obtained.

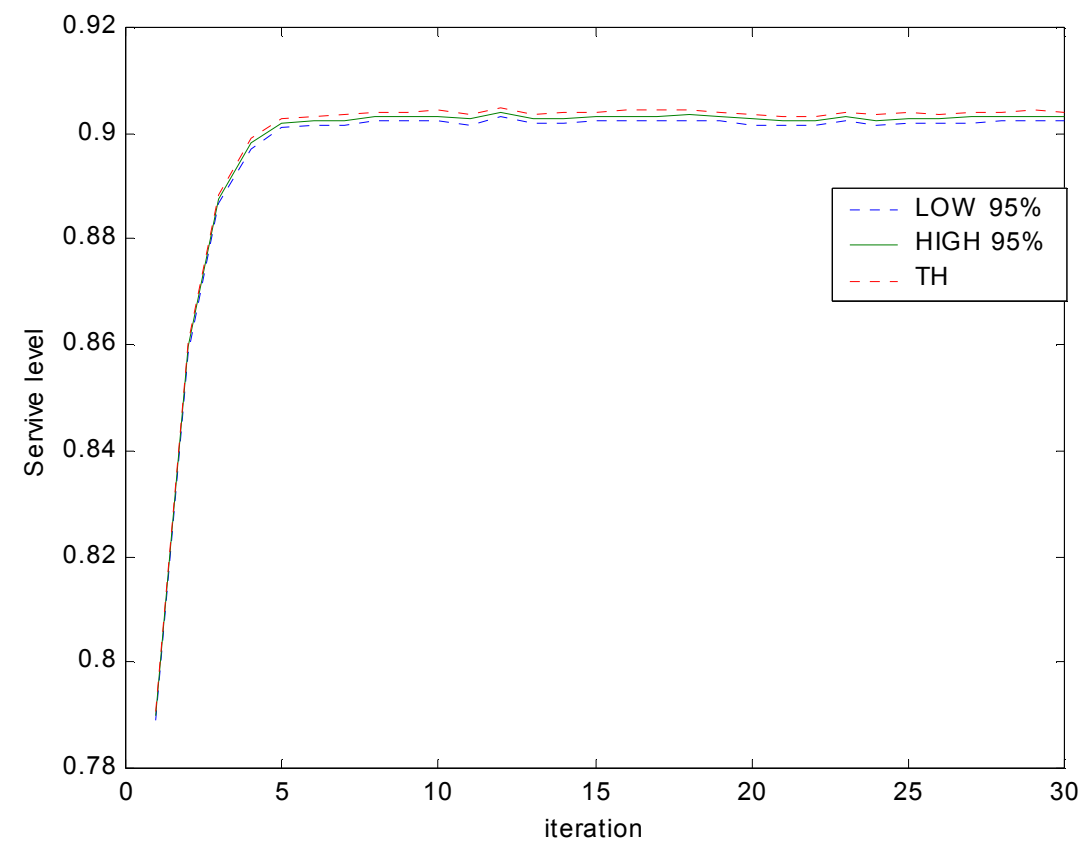

Figure 10. Simulation results

\section{Concluding Remarks}

In this study, we present an updating mechanism for the order-up-to level in a periodic review inventory system with lost sales. The method utilizes only the POS data and operates in the absence of any demand and inventory information. The method yields the desired service level even starting with very low service levels after a few iterations.

Embedding the observations on the system's performance into the inventory control mechanism yields a closed-loop control as opposed to other approaches where the inventory control parameters are not changed once they are set. The results presented in this study demonstrate that 
the closed-loop control approach to inventory management with unobserved lost sales delivers very satisfactory performance.

If plausible, the dynamic parameter-updating scheme presents a number of benefits, its simplicity being the foremost one. For example, a retailer that carries thousands of stock keeping units (SKUs) can automatically manage inventories of certain SKUs without risking holding too much or too little inventory, or without too much deviation from a pre-specified service level. Another important benefit of the periodic updating scheme is its operation without using any inventory information. This property of the periodic updating scheme widens its applicability, because one need not collect inventory information to implement the proposed procedure. Moreover, when the periodic updating scheme converges to the desired service level, the parameters of the actual demand process can be readily determined.

The updating mechanism uses POS information in equal length intervals. Most of the data collection systems used at retailers have the capability of collecting and storing transaction data. However, storing transaction information over a period of time requires massive data storage capacity. Furthermore, this may not be necessary for the purpose of inventory control. Increasing the length of the POS data collection interval reduces the data storage requirements of the data warehouse considerably. The simulation study shows that even using hourly POS data allows one to manage the inventory satisfactorily. Determining the temporal granularity of POS data according to the objectives of the management is an important implementation problem to manage the resources of data warehouses effectively.

One of the important extensions of this methodology will be to develop a similar updating mechanism for products with non-stationary demand. The non-stationarity of the demand may be caused by the hourly, weekly, and seasonal arriving and purchasing patterns of customers and also by marketing activities such as promotions. In principle, if we assume that the forecast error between the time-series used to describe the non-stationary demand and the observations is normally distributed, the proposed method can be applied directly. Similarly, if the effect of marketing activity can be prescribed beforehand, this information can be used to transform the POS data into a stationary series. These transformations allow us to use the methodology presented in this study for products with non-stationary demand. A detailed study of the non-stationary case is left for future research.

Another important extension will be to include the substitution effects. However, understanding the substitution effects require more information about not only the inventory level of the product that is being analyzed but also the inventory levels of its possible substitutes. This extension is also left for future research.

\section{Acknowledgement}

The authors thank the Associate Editor and an anonymous referee for their helpful comments. 


\section{References}

Agrawal, N.S. and S.A. Smith (1996), "Estimation Methods for Retail Inventory Management with Unobservable Lost Sales," Naval Research Logistics, Vol. 43, pp. 839-861.

Anupindi, R., M. Dada, and S. Gupta, (1998), "Estimation of Consumer Demand with Stockout Based Substitution: An Application to Vending Machine Products," Marketing Science, Vol. 17, No. 4, pp. 406-423.

Bell, P.C. (2000), "Forecasting Demand Variation When There are Stockouts," Journal of the Operational Research Society, Vol. 51, pp. 358-363.

Buzacott, J.A., (1999), "Dynamic Inventory Targets Revisited," Journal of the Operational Research Society, Vol. 50, pp. 697-703.

Chen, F., Z. Drezner, J.K. Ryan, and D. Simchi-Levi, (2000), "Quantifying the Bullwhip Effect in a Simple Supply Chain: The Impact of Forecasting, Lead Times and Information," Management Science, Vol. 46, No. 3. pp. 436-443.

East, R., W. Lomax, G. Willson, and P. Harris (1994), "Decision Making and Habit in Shopping Times," European Journal of Marketing, Vol. 28, No. 4, pp. 56-71.

Hopp, W. J., M. L. Spearman, and R. Q. Zhang, "Easily Implementable Inventory Control Policies," Operations Research, Vol. 45, No. 3, pp. 327-340.

Kahn, B.E. and D.C. Schmittlein (1989), "Shopping Trip Behavior: An Empirical Investigation," Marketing Letters, Vol. 1, No. 1, pp. 55-69.

Lam, S.Y., M. Vandenbosch, and M. Pearce (1998), "Retailer Sales Force Scheduling Based on Store Traffic Forecasting," Journal of Retailing, Vol. 74, No. 1, pp. 61-88.

Lariviere, M. A. and E. L. Porteus (1999), "Stalking Information: Bayesian Inventory Management with Unobserved Lost Sales," Management Science, Vol. 45, No. 3, pp. 346-363.

Lau, H-S., and A. H-L. Lau, (1996), "Estimating the Demand Distributions of Single-Period Items Having Frequent Stockouts," European Journal of Operational Research, Vol. 92, pp. 254-265.

Nahmias, S. (1994), "Demand Estimation in Lost Sales Inventory Systems," Naval Research Logistics, Vol. 41, pp. 739-757.

Silver, E. A., D. F. Pyke, and R. Peterson, Inventory Management and Production Planning and Scheduling, 1998, John Wiley and Sons, New York.

Walters, R. G. and H.J. Rinne, (1986), “Am Empirical Investigation into the Impact of Price Promotions on Retail Store Performance,” Journal of Retailing, Vol. 62, No. 3, pp. 237-265.

\section{Appendix}

\section{Proof of Proposition 1:}

Let $\delta_{i}$ be an indicator variable which is 1 if the demand in the POS data collection period $i$ is smaller than the starting inventory level of the data collection period $i$, and 0 otherwise. Then Equation (1) can be rewritten as 


$$
\hat{\mu}_{t}=\frac{1}{N} \sum_{i=1}^{N}\left(s_{i} \boldsymbol{\delta}_{i}+s_{i}\left(1-\boldsymbol{\delta}_{i}\right)\right)
$$

If we had perfect information about the shelf inventory level in POS data collection period $i$, then we would be able to determine the $\boldsymbol{\delta}_{t}$ values, and estimate the mean of the demand per POS data collection period with $\tilde{\mu}_{t}$ as

$$
\tilde{\mu}_{t}=\frac{\sum_{i=1}^{N} s_{i} \boldsymbol{\delta}_{i}}{\sum_{i=1}^{N} \boldsymbol{\delta}_{i}} .
$$

In other words, we would only use the sales data when the ending inventory level of a POS data collection period is not zero to estimate the mean of the demand. Note that when the beginning inventory of a POS data collection period is positive and the realized demand of the period is greater than the available units in the inventory, the excess demand of the period is lost. In this case, although $s_{i}$ is positive, it would not reflect the actual demand of the period. Let $n_{R}$ be the number of times the inventory is replenished during $[t-1, t)$. That is, $n_{R}$ is the largest integer smaller than the duration of the $[t-1, t)$ time interval divided by $R$. The underestimation of demand, however, can be experienced in at most $n_{R}$ POS data collection periods, because in each inventory review period, the inventory level may become zero in at most one POS data collection period. Since $n_{R}$ is assumed to be much smaller than $N$, we can assume that the sales of such period are equal to zero. Under this approximation, Equation (31) can be written as

$$
\hat{\boldsymbol{\mu}}_{t}=\frac{1}{N} \sum_{i=1}^{N} s_{i} \boldsymbol{\delta}_{i}=\tilde{\mu}_{\mathrm{t}} \frac{\sum_{i=1}^{N} \boldsymbol{\delta}_{i}}{N} .
$$

When the length of the POS data collection period is small, the ratio $\sum_{i=1}^{N} \boldsymbol{\delta}_{i} / N$ is known as the Ready Rate (see Silver et al., Chapter 7) and is equal to the fraction of demand satisfied from inventory under Poisson demand. Hopp et al. (1997) approximate the ready rate of an inventory system with the fraction of demand that is met from inventory and argue that the approximation is reasonably good when the desired service level is high. Then by approximating the fraction of demand satisfied from inventory with the fraction of time the inventory is greater than zero, Equation (33) can be rewritten as

$$
\hat{\mu}_{t} \cong \tilde{\mu}_{t} \beta_{t-1} \text {. }
$$

Similarly, the estimate of the standard deviation of demand, $\tilde{\sigma}_{t}$, based on the periods where the inventory is greater than the demand, i.e., the sales and the demand are equal, is obtained as 


$$
\tilde{\boldsymbol{\sigma}}_{t}=\sqrt{\frac{1}{\sum_{i=1}^{N} \boldsymbol{\delta}_{i}-1} \sum_{i=1}^{N}\left(s_{i}-\tilde{\mu}_{t}\right)^{2} \boldsymbol{\delta}_{i}} .
$$

For large $N, \sum_{i=1}^{N} \delta_{i}-1 \cong \beta_{t-1} N-1$ that yields

$$
\tilde{\boldsymbol{\sigma}}_{t}^{2}=\sum_{i=1}^{N} s_{i}^{2} \frac{\boldsymbol{\delta}_{i}}{\beta_{t-1} N-1}-\tilde{\mu}_{t}^{2}
$$

Rewriting Equation (2) yield

$$
\hat{\sigma}_{t}^{2}=\sum_{i=1}^{N} \frac{s_{i}^{2}}{N-1}-2 \hat{\mu}_{t} \sum_{i=1}^{N} \frac{s_{i}}{N-1}+\hat{\mu}_{t}^{2} \frac{N}{N-1} .
$$

Now replacing $\sum_{i=1}^{N} s_{i}^{2}=\sum_{i=1}^{N} s_{i}^{2} \delta_{i}=\left(\tilde{\boldsymbol{\sigma}}_{t}^{2}+\tilde{\mu}_{t}^{2}\right)\left(\beta_{t-1} N-1\right)$ in Equation (37) yields

$$
\hat{\sigma}_{t}^{2}=\tilde{\sigma}_{t}^{2}\left[\frac{\beta_{t-1} N-1}{N-1}\right]+\tilde{\mu}_{t}^{2} \beta_{t-1}\left(1-\beta_{t-1}\right) \frac{N}{N-1} .
$$

Finally, approximating $N-1$ by $N$ for large $N$ yields

$$
\hat{\sigma}_{t}^{2} \cong \tilde{\sigma}_{t}^{2} \beta_{t-1}+\tilde{\mu}_{t}^{2} \beta_{t-1}\left(1-\beta_{t-1}\right) \text {. }
$$

If we have sufficient data, $\mu$ and $\sigma$ are equivalent to $\tilde{\mu}_{t}$ and $\tilde{\sigma}_{t}$, respectively. Now replacing $\tilde{\mu}_{t}$ and $\tilde{\sigma}_{t}$ with $\mu$ and $\sigma$ in Equations (34) and (39) yield the results presented in Equations (3) and (4).

\section{Proof of Proposition 2:}

a. The first proposition states that the desired service level $\beta$ is a solution of $x=g(x)$. Suppose $\beta_{t-1}=\beta$ for some $t=T>1$. Then substituting $\beta_{t-1}=\beta$ in equations (7) and (8) shows that the estimates of the mean and standard deviation of demand match the true mean and standard deviation of demand. Since $\beta$ is very high in practice, the standard assumption on the accuracy of Equation (12) stated in Section 3.4 allows us to state that $\beta$ is a solution of $x=g(x)$.

b. For small values of $\beta_{t}$ closer to zero, the actual expected number of units short will be close to $\mu R$ following Equation (15), $E\left[I_{1}\right]$ will be close to zero following Equation (24), i.e., the expected order size will be $S_{t}$, and the realized but unobserved service level will be approximately 


$$
\beta_{t} \cong \frac{S_{t}}{S_{t}+\mu R}
$$

For $\beta_{t-1}=0$, the order-up-to level that will be set in the next update will be 0 , then the next service level will be 0 ,i.e, $g(0)=0$.

\section{Proof of Proposition 3:}

The function $g\left(\beta_{t-1}\right)$ is an increasing function of $\beta_{t-1}$ if

$$
\frac{d \boldsymbol{\beta}_{t}}{d \boldsymbol{\beta}_{t-1}}=\frac{d}{d \boldsymbol{\beta}_{t-1}} \frac{E[Q]}{E[Q]+E\left[N_{S}\right]}=\frac{d}{d S_{t}} \frac{E[Q]}{E[Q]+E\left[N_{S}\right]} \cdot \frac{d S_{t}}{d \boldsymbol{\beta}_{t-1}}>0
$$

Now, differentiating $E[Q]$ with respect to $S_{t}$ by using the Leibniz's rule yields

$$
\begin{aligned}
\frac{d E[Q]}{d S_{t}} & =\frac{d}{d S_{t}}\left(S_{t}-\int_{0}^{S_{t} S_{t}-y} \int_{0}\left(S_{t}-y-z\right) f_{Z}(z) f_{Y}(y) d z d y\right) \\
& =1-\int_{0}^{S_{t} S_{t}-y} \int_{0} f_{Z}(z) f_{Y}(y) d z d y>0
\end{aligned}
$$

Similarly, differentiating $E\left[N_{s}\right]$ with respect to $S_{t}$ gives

$$
\begin{aligned}
\frac{d E\left[N_{S}\right]}{d S_{t}}= & \frac{d}{d S_{t}}\left(\int_{0}^{S_{t}} \int_{S_{t}-y}^{\infty}\left(x+y-S_{t}\right) f_{X}(x) f_{Y}(y) d x d y+\mu R \Phi\left(\frac{S_{t}-\mu L}{\sigma \sqrt{L}}\right)\right) . \\
& =-\int_{0}^{S_{t}} \int_{S_{t}-y}^{\infty} f_{X}(x) f_{Y}(y) d x d y<0
\end{aligned}
$$

Now,

$$
\frac{d}{d S_{t}} \frac{E[Q]}{E[Q]+E\left[N_{S}\right]}=\frac{d E[Q]}{d S_{t}} \frac{E\left[N_{S}\right]}{\left(E[Q]+E\left[N_{S}\right]\right)^{2}}-\frac{d E\left[N_{S}\right]}{d S_{t}} \frac{E[Q]}{\left(E[Q]+E\left[N_{S}\right]\right)^{2}}>0
$$

Since, $\frac{d E\left[N_{S}\right]}{d S_{t}}<0, \frac{d E[Q]}{d S_{t}}>0, E\left[N_{s}\right]>0$, and $E[Q]<0$, the function $g\left(\beta_{t-1}\right)$ is an increasing function of $\beta_{t-1}$ if

$$
\frac{d S_{t}}{d \beta_{t-1}}=\frac{d}{d \beta_{t-1}}\left(\mu \frac{\beta_{t-1}}{\beta}(R+L)+z_{t} \sigma \frac{\sqrt{R+L}}{\beta c v} \sqrt{\beta_{t-1} \beta\left(c v^{2}+1\right)-\beta_{t-1}^{2}}\right)>0
$$

where 


$$
z_{t}=\eta^{-1}\left(\frac{R(1-\beta)}{\beta \sqrt{R+L}} \frac{\sqrt{\beta_{t-1}}}{\sqrt{\beta\left(c v^{2}+1\right)-\beta_{t-1}}}\right)
$$

By using $\frac{d \eta^{-1}(z)}{d z}=-\frac{1}{\Phi\left(\eta^{-1}(z)\right)}$, the above equation can be written as

$$
\frac{d S_{t}}{d \beta_{t-1}}=\frac{\mu \sqrt{R+L}}{\beta}\left(\sqrt{R+L}+\frac{k \eta\left(z_{t}\right)\left(\eta\left(z_{t}\right)+\Phi\left(z_{t}\right)\right)}{\Phi\left(z_{t}\right)}\left[\frac{\Phi\left(z_{t}\right)-\eta\left(z_{t}\right)}{\eta^{2}\left(z_{t}\right)\left(\eta\left(z_{t}\right)+\Phi\left(z_{t}\right)\right)}-\frac{1}{k^{2}}\right]\right)
$$

where

$$
k=\frac{R(1-\beta)}{\beta \sqrt{R+L}} .
$$

The sign of $\frac{d S_{t}}{d \beta_{t-1}}$ depends on the sign of the term inside the parentheses. The firs term $\sqrt{R+L}$ is always positive. The sign of the second term depends on the term inside the brackets. It can be shown that the function $\frac{\Phi\left(z_{t}\right)-\eta\left(z_{t}\right)}{\eta^{2}\left(z_{t}\right)\left(\eta\left(z_{t}\right)+\Phi\left(z_{t}\right)\right)}$ is a monotone increasing function of $z_{t}$. Therefore the equation $\frac{\Phi\left(z_{t}\right)-\eta\left(z_{t}\right)}{\eta^{2}\left(z_{t}\right)\left(\eta\left(z_{t}\right)+\Phi\left(z_{t}\right)\right)}=\frac{1}{k^{2}}$ has a unique solution. That is, the term inside the brackets changes sign only once.

Even though the term inside the brackets can take negative values, the term inside the parentheses can always be positive depending on the system parameters. Then $\frac{d S_{t}}{d \beta_{t-1}}$ changes its sign at most once. Since the functions $\Phi(\cdot)$ and $\eta(\cdot)$ are defined and this point can be determined numerically.

Let $z_{t}^{*}$ be the value of $z_{t}$ where $\frac{d S_{t}}{d \beta_{t-1}}=0$ if it exists. Once $z_{t}^{*}$ is determined, the corresponding service level for this value of $z_{t}$ is evaluated from Equation (42). Let the specific value of $\beta_{t-1}$ that satisfies Equation (42) with $z_{t}=z_{t}^{*}$ be $\beta^{\prime}$. Then either $\frac{d S_{t}}{d \beta_{t-1}}>0$ for all $\beta_{t-1}$, or it changes at a single point $x=\beta^{\prime}$ and $\frac{d S_{t}}{d \beta_{t-1}} \geq 0$ for $\beta^{\prime} \geq \beta_{t-1}>0$ and $\frac{d S_{t}}{d \beta_{t-1}}<0$ for $\beta^{\prime}<\beta_{t-1}$.

As a result $g\left(\beta_{t-1}\right)$ is either an increasing function of $\beta_{t-1}$ for all $\beta_{t-1}$, or it is an increasing function for $\beta^{\prime} \geq \beta_{t-1}>0$ and decreasing function $\beta \geq \beta_{t-1}>\beta^{\prime}$. 


\section{Proof of Proposition 4:}

The proofs of (a) and (b) follow directly the one given in Property 2.

c. In order to show that $\frac{d h\left(\beta_{t-1}\right)}{d \beta_{t-1}} \geq 0$ for $\beta^{\prime} \geq \beta_{t-1}>0, h\left(\beta_{t-1}\right)$ is differentiated in each region separately:

In region $\mu(R+L)<S_{t}$ is;

$$
\frac{d h\left(\beta_{t-1}\right)}{d \beta_{t-1}}=\frac{d}{d \beta_{t-1}} \frac{\mu R}{\mu R+\sigma \sqrt{R+L} \eta\left(\underline{z}_{t}\right)}=\frac{\mu R \Phi\left(\underline{z}_{t}\right)}{\left(\mu R+\sigma \sqrt{R+L} \eta\left(\underline{z}_{t}\right)\right)} \cdot \frac{d S_{t}}{d \beta_{t-1}} .
$$

In region $\mu(R-L) \leq S<\mu(R+L)$,

$$
\frac{d h\left(\beta_{t-1}\right)}{d \beta_{t-1}}=\frac{d}{d \beta_{t-1}} \frac{S_{t}+\mu(R-L)}{S_{t}+\mu(R-L)+2 \sigma \sqrt{R+L} \eta\left(\underline{z}_{t}\right)}=\frac{\sigma \sqrt{R+L} \eta\left(\underline{z}_{t}\right)+\left(S_{t}+\mu(R-L)\right) \Phi\left(\underline{z}_{t}\right)}{2\left(S_{t}+\mu(R-L)+2 \sigma \sqrt{R+L} \eta\left(\underline{z}_{t}\right)\right)^{2}} \cdot \frac{d S_{t}}{d \beta_{t-1}}
$$

and in region $S_{t}<\mu(R-L)$ is

$$
\frac{d h\left(\beta_{t-1}\right)}{d \beta_{t-1}}=\frac{d}{d \beta_{t-1}} \frac{S_{t}}{S_{t}+\sigma \sqrt{R+L} \eta\left(\underline{z}_{t}\right)}=\frac{\sigma \sqrt{R+L} \eta\left(\underline{z}_{t}\right)+S_{t} \Phi\left(\underline{z}_{t}\right)}{\left(S_{t}+\sigma \sqrt{R+L} \eta\left(\underline{z}_{t}\right)\right)^{2}} \cdot \frac{d S_{t}}{d \beta_{t-1}} .
$$

Considering the above expressions for each region, $\frac{d h\left(\beta_{t-1}\right)}{d \beta_{t-1}}>0$, if $\frac{d S_{t}}{d \beta_{t-1}}>0$. The sign of $\frac{d S_{t}}{d \beta_{t-1}}$ is investigated in the proof of Proposition 3.

Following the result in Proposition 3 , either $\frac{d h\left(\beta_{t-1}\right)}{d \beta_{t-1}} \geq 0$ for all $\beta_{t-1}$, or $\frac{d h\left(\beta_{t-1}\right)}{d \beta_{t-1}} \geq 0$ for $\beta^{\prime} \geq \beta_{t-1}>0$ and $\frac{d h\left(\beta_{t-1}\right)}{d \beta_{t-1}}<0$ for $\beta>\beta_{t-1}>\beta^{\prime}$

d. By differentiating the above results for each region, it can be shown that $\frac{d^{2} h\left(\beta_{t-1}\right)}{d \beta_{t-1}^{2}}<0$, if $\frac{d^{2} S_{t}}{d \beta_{t-1}^{2}}>0$. Differentiating Equation (43) yields 


$$
\begin{aligned}
& \frac{d^{2} S_{t}}{d \beta_{t-1}^{2}}= \\
& \frac{\mu R(1-\beta)}{\beta^{2}} \frac{\eta^{2}\left(\underline{z}_{t}\right) \phi\left(\underline{z}_{t}\right)+\Phi^{2}\left(\underline{z}_{t}\right)\left(2 \eta\left(\underline{z}_{t}\right)+\Phi\left(\underline{z}_{t}\right)\right)}{\Phi^{2}\left(\underline{z}_{t}\right)}\left[\frac{1}{k^{2}}-\frac{\Phi^{3}\left(\underline{z}_{t}\right)-\eta^{2}\left(\underline{z}_{t}\right) \phi\left(\underline{z}_{t}\right)}{\eta^{2}\left(\underline{z}_{t}\right)\left(\eta^{2}\left(\underline{z}_{t}\right) \phi\left(\underline{z}_{t}\right)+\Phi^{2}\left(\underline{z}_{t}\right)\left(2 \eta\left(\underline{z}_{t}\right)+\Phi\left(\underline{z}_{t}\right)\right)\right)}\right]
\end{aligned}
$$

Similar to the case in Property 3 , the term inside the brackets changes sign at a unique value of $\underline{z}_{t}$ that corresponds to a unique service level via Equation (42). Let this value be $\beta^{\prime \prime}$. Then $\frac{d^{2} h\left(\beta_{t-1}\right)}{d \beta_{t-1}^{2}}<0$ for if $x<\beta^{\prime \prime}$, and $\frac{d^{2} h\left(\beta_{t-1}\right)}{d \beta_{t-1}^{2}}>0$ for if $x>\beta^{\prime \prime}$.

\section{Proof of Proposition 5:}

Since $h(x)$ is a lower bound of $g(x), g(0)=h(0)=0$, and $g(\beta)=h(\beta)=\beta$, showing that $h(x)$ is greater than $x$ in the interval $0<x<\beta$ is sufficient to show the convergence of the updating mechanism to the desired service level.

The properties of $g(x)$ and $h(x)$ include two specific values, $\beta^{\prime}$ and $\beta^{\prime \prime}$, that are determined by the parameters of a given system. Instead of using their numerical values, we can consider the ordinal placement of $\beta, \beta^{\prime \prime}$, and $\beta$.

For $0<\beta<1$, considering the ordinal placement of $\beta, \beta^{\prime \prime}$, and $\beta$ there are six different cases: 1. $\beta^{\prime \prime} \leq \beta \leq \beta, \quad 2 . \beta \leq \beta^{\prime \prime} \leq \beta, \quad 3 . \beta \leq \beta \leq \beta^{\prime \prime}, \quad 4 . \quad \beta \leq \beta^{\prime \prime} \leq \beta, \quad$ 5. $\beta \leq \beta \leq \beta^{\prime \prime}, 6 . \beta^{\prime \prime} \leq \beta \leq \beta$. We can study the implications of each ordering separately on the convergence of the updating mechanism.

In cases 1,2 , and $3, \beta$ is greater than or equal to $\beta$. Therefore $h(x)$ increases in the region $\left[0, \beta^{\prime}\right]$ and decreases in the region $\left[\beta^{\prime}, \beta\right]$. Since $h(\beta)=\beta$ (Proposition 1a), the level it reaches at $x=\beta^{\prime}$ must be greater than or equal to $\beta$ in order to reach $h(\beta)=\beta$ without increasing, i.e., $h\left(\beta^{\prime}\right) \geq \beta$. Since $h(x)$ is also concave in $\left[0, \beta^{\prime}\right], h(x)$ must be greater than $x$ for $0<x<\beta$.

In cases 4 and 5 , the critical points $\beta$ and $\beta^{\prime \prime}$ are greater than or equal to $\beta$. This means that $h(x)$ is a concave increasing function for $0<x<\beta$. Since $h(0)=0, h(x)$ is greater than $x$ for $0<x<\beta$.

In case $6, \beta^{\prime \prime}<\beta<\beta, h(x)$ increases first with a decreasing trend and then with a nondecreasing trend until it reaches $h(\beta)=\beta$. Since $h(0)=0$, in both of these regions, the function $h(x)$ lies above $x$, i.e., $h(x)>x$ for $0<x<\beta$.

We established that $h(x)>x$ for $0<x<\beta$. Therefore $g(x)>x, 0<x<\beta$. As a result $\beta_{t+1}=g\left(\beta_{t}\right)>\beta_{t}$, $0 \leq \beta_{t}<\beta$ for $t=1,2 \ldots$. Thus the updating mechanism converges to a unique point which is the desired service level. 